\title{
Anpassungen der technischen Infrastruktur im Siedlungswasserbau
}

Die Hauptbauphasen der Infrastruktur im Bereich des Siedlungswasserbaus für Wasserversorgungsnetze, Kanalisationen und Kläranlagen liegen entsprechend den Aufzeichnungen der Kommunalkredit Public Consulting (KPC) in den 1960er- bis 1990er-Jahren. Derzeit existieren in Österreich knapp 80.000 km Wasserleitungsinfrastruktur und $90.000 \mathrm{~km}$ Kanalisation. Seit Anfang der 2000er-Jahre hat sich der Fokus vom Neubau weitgehend auf die Erhaltung und regelmäßige Anpassung der Infrastruktur auf die sich ändernden Rahmenbedingungen verlagert. Da Anpassungsmaßnahmen im Infrastrukturbereich erfahrungsgemäß zum Teil eine sehr lange Vorlaufzeit haben, kommt der langfristigen - strategischen - Planung eine zunehmend wichtiger werdende Rolle zu. Verfügbares Wissen und Daten werden systematisch in Informationen verwandelt, die es den EntscheidungsträgerInnen ermöglichen, rechtzeitig die richtigen Entscheidungen zu treffen, um die übergeordneten und langfristigen Ziele bestmöglich verfolgen zu können.

\section{Strategische Planung in der Trinkwasserversorgung}

Durch die sehr große gesellschaftliche Bedeutung von Trinkwasser und den immer höher werdenden Nutzungsdruck auf Wasserressourcen ergeben sich stetig steigende Anforderungen an die Trinkwasserversorgung bezüglich Qualität, Sicherung und Verfügbarkeit. Um langfristig die Qualitätsstandards sowie die ökologische und ökonomische Nachhaltigkeit des Unternehmens und von Arbeitsprozessen noch zu erhöhen, werden bereits unterschiedliche Qualitätssicherungsinstrumente und Strategien angewendet.

Eine längerfristige Planung zur Optimierung (Strategie) berücksichtigt auch eine technische Nachhaltigkeit, um eine langfristig gesicherte und strukturell zweckmäßige Trinkwasserversorgung zu gewährleisten. Dabei müssen übergeordnete Ziele und Rahmenbedingungen bei der Planung berücksichtigt und gegebenenfalls immer wieder neu beurteilt werden.

Die übergeordneten und langfristigen Ziele können folgendermaßen zusammengefasst werden:

- Die Qualität des Trinkwassers ist durch geeignete Anlagen, Verfahren, Maßnahmen und geschultes Fachpersonal sicherzustellen (Lebensmittelsicherheit, Produktqualität).

- Die Verfügbarkeit von genügend geeignetem Rohwasser ist - unter Berücksichtigung möglicher Nutzungskonflikte mit anderen, z. B. der Landwirtschaft - langfristig sicherzustellen (Versorgungssicherheit).

- Der Werterhalt der Anlagen ist durch einen gezielten Unterhalt und eine langfristig gesicherte Finanzierung zu gewährleisten (Versorgungssicherheit, Löschwasser).

- Die Anliegen des Natur- und Umweltschutzes und damit insbesondere auch des Gewässerschutzes sind unter Beachtung der Priorität der Wasserversorgung zu berücksichtigen (Nachhaltigkeit).

- Den Anliegen der KonsumentInnen ist durch Informationen und Transparenz bei den Kosten Rechnung zu tragen (Kundlnnenzufriedenheit).

- Ein wirtschaftlicher Betrieb ist durch eine geeignete Form der Organisation und durch eine optimale Größe der Infrastrukturen sicherzustellen (Wirtschaftlichkeit).

Die Grundlagen der strategischen Planung stellen Daten und Informationen dar, auf Basis derer Entscheidungen abgeleitet werden können. Diese erfolgt unter Berücksichtigung der Unsicherheit, der Flexibilität und der Anpassungserfordernisse sowie der Anforderungen und Methoden für den modernen Anlagenbetrieb. Neben der Werterhaltung und Optimierung sind aber auch Innovationen, z. B. bei 
der Anwendung neuer Messmethoden, bei der Onlineüberwachung oder der Anwendung der numerischen Simulation etc. notwendig. Diese ermöglichen ein verbessertes Prozessverständnis und die Simulation möglicher Anpassungen. Die jeweiligen Datenerfordernisse ergeben sich aus Fragestellung und Umfang der strategischen Planung und können Datengrundlagen zur Beurteilung von gesamten Unternehmen (z. B. Unternehmensbenchmarking) bis hin zur Optimierung von Anlagen und Prozessen zur Qualitäts- bzw. Struktur- und Prozesssicherung umfassen.

Die vorliegende Ausgabe der Österreichischen Wasser- und Abfallwirtschaft spannt einen weiten Bogen über die Themenbereiche der strategischen Planung in der Trinkwasserversorgung vom Einzugsgebiet bis zum/zur Endverbraucher/in. Beginnend bei den sich verändernden Rahmenbedingungen der Versorgung werden neue Möglichkeiten der Risikobeurteilung und im Ressourcenmonitoring bei Brunnen und Quellen betrachtet. Anhand konkreter Anwendungsfälle werden der Einsatz moderner Messtechnik sowie die Integration der numerischen Modellierung in der strategischen Planung dargestellt. Weiters werden die Einflüsse auf die Rehabilitationsplanung bei Erneuerungsmaßnahmen im Wasserleitungsnetz dargelegt und die Möglichkeiten der betrieblichen Störfallvorsorge und im Umgang mit einem Störfall (Notfall, Krise) anhand der Umsetzung in der Steiermark beleuchtet.

\section{Univ.-Prof. DI Dr. T. Ertl (西) •}

Priv.-Doz. DI Dr. R. Perfler ( $₫)$

Department Wasser, Atmosphäre, Umwelt, Institut für Siedlungswasserbau, Industriewasserwirtschaft und Gewässerschutz, Universität für Bodenkultur Wien,

Muthgasse 18,

1190 Wien, Österreich

thomas.ert|@boku.ac.at;

reinhard.perfler@boku.ac.at 